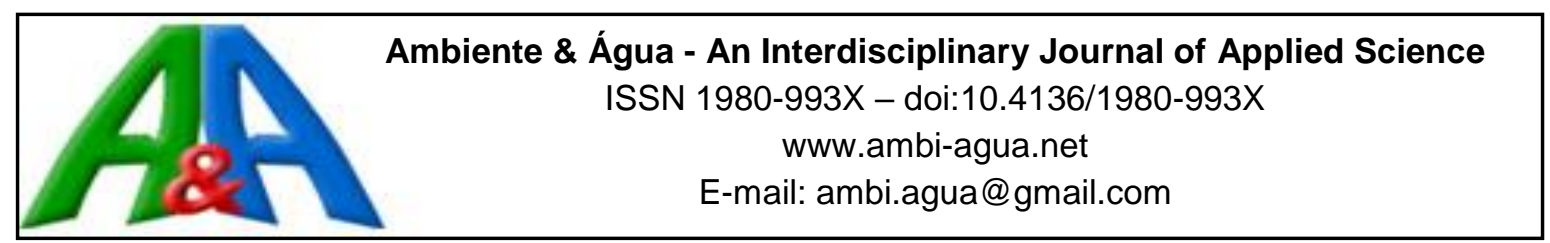

\title{
Efeito do dióxido de carbono e da radiação solar na biomassa algal produzida em fotobiorreator laminar
}

\author{
doi:10.4136/ambi-agua.1877
}

Received: 01 Mar. 2016; Accepted: 14 May 2016

\author{
Tiago Borges Ferreira ${ }^{1 *}$; Jesús Fernández González ${ }^{2}$; \\ André Luís Teixeira Fernandes ${ }^{3}$ \\ ${ }^{\mathbf{1}}$ Universidade de São Paulo (USP), São Carlos, SP, Brasil \\ Departamento de Hidráulica e Saneamento \\ ${ }^{2}$ Universidad Politécnica de Madrid (UPM), Madrid, España \\ Escuela Técnica Superior de Ingenieros Agrónomos \\ ${ }^{3}$ Universidade de Uberaba (UNIUBE), Uberaba, MG, Brasil \\ *Autor correspondente: e-mail: tiago_bferreira@hotmail.com, \\ j.fernandez@upm.es, andre.fernandes@uniube.br
}

\section{RESUMO}

O presente trabalho teve como objetivo mensurar a influência do fornecimento artificial de $\mathrm{CO}_{2}$ e da variação da radiação fotossinteticamente ativa na biomassa algal em um fotobiorreator laminar. Utilizou-se para tanto, avaliações de produção e concentração de nitrogênio e fósforo na biomassa coletada semanalmente em cada uma das 36 subdivisões do sistema. Além disso, se determinou, por meio de cálculos baseados na produtividade, os intervalos de eficiências produtiva e energética. $\mathrm{O}$ fornecimento artificial de $\mathrm{CO}_{2}$ influenciou a produção de biomassa, incrementando a produtividade de 3,9 a $27,0 \%$. A radiação fotossinteticamente ativa manteve estreita relação com a produção de biomassa, com variação de até $60,5 \%$, produzindo entre 1,42 e $2,28 \mathrm{~g} \mathrm{~m}^{-2} \mathrm{~d}^{-1}$. A concentração de nitrogênio na biomassa não foi influenciada por nenhum dos fatores avaliados, porém o fósforo sofreu influência de ambos os fatores. As eficiências produtiva e energética tiveram as mesmas influências, variando de 0,28 a $0,44 \mathrm{~g} \mathrm{~mol}^{-1}$ e 2,12 a $3,34 \%$, respectivamente.

Palavras chave: Chlorella, eficiência energética, microalgas.

\section{Effect of carbon dioxide and sunlight on algae biomass produced in laminar photobioreactor}

\begin{abstract}
This work measured the influence of $\mathrm{CO}_{2}$ supply and photosynthetically active radiation on microalgae production in the laminar photobioreactor. In order to accomplish this, the production and concentration of nitrogen and phosphorus in the biomass collected from each of the 36 subdivisions of the system was analyzed weekly. Additionally, productive and energetic efficiencies were determined by estimating biomass production. The artificial $\mathrm{CO}_{2}$ supply affected biomass production, increasing the productivity from 3.9 to $27.0 \%$. The photosynthetically active radiation was related to the production of biomass, which varied up to $60.5 \%$, producing between 1.42 and $2.28 \mathrm{~g} \mathrm{~m}^{-2} \mathrm{~d}^{-1}$. The nitrogen concentration in the
\end{abstract}


biomass was not influenced by any of the evaluated factors; nevertheless, the phosphorus was influenced by both factors. The productive and energetic efficiencies had similar influence, ranging from 0.28 to $0.44 \mathrm{~g} \mathrm{~mol}^{-1}$ and from 2.12 to $3.34 \%$, respectively.

Keywords: Chlorella, energetic efficiency, microalgae.

\section{INTRODUÇÃO}

As microalgas são consideradas como a origem dos fitoplanctons marinhos e dos vegetais superiores, que em conjunto são responsáveis pela presença de oxigênio na atmosfera. Porém, sua primeira utilização pelos seres humanos foi somente há 2000 anos, quando a espécie Nostoc sp. foi utilizada como alimento na China (Priyadarshani e Rath, 2012).

Atualmente, o cultivo de microalgas tem ganhado destaque, principalmente por apresentar diversas vantagens econômicas e ambientais frente a outros cultivos, dentre as quais se destacam: potencial para produzir mais biocombustível que qualquer outra fonte oleaginosa (Chen et al., 2009); não compete com a agricultura tradicional, por ser geralmente cultivada em fotobiorreatores, utilizando áreas não agricultáveis (Chen et al., 2009; Soares et al., 2011); pode-se cultivar em diversos climas e condições hídricas, além de utilizar dióxido de carbono $\left(\mathrm{CO}_{2}\right)$ de diversas fontes (Chen et al., 2009; Priyadarshani e Rath, 2012).

Sua composição permite a extração de ampla gama de produtos, incluindo fármacos, cosméticos, vitaminas, minerais, óleos essenciais, proteína e substâncias bioativas, os quais são destinados principalmente para a produção de alimentos, rações animais e biofertilizantes (Benemann, 2006; Borghetti, 2009; Chen et al., 2009; Mata et al., 2010).

Cultivadas inicialmente somente em reatores abertos, alcançavam baixas produções e não se possibilitava o controle das condições de cultivo. Por estas limitações, estimulou-se o desenvolvimento dos modernos sistemas, subdivididos em dois grandes grupos; fechados e híbridos, que garantiram maior controle do cultivo e a intensificação da produção. O sistema em questão é um híbrido, patenteado pela Universidade Politécnica de Madri como fotobiorreator laminar (do inglês-PBRL) sob o $\mathrm{n}^{\circ}$ EP2568038 A1 (Fernández, 2011). O modelo é constituído de duas placas de geotêxtil verticais, formando uma câmara vazia em seu interior, na qual se introduz o fornecimento artificial de $\mathrm{CO}_{2}$, e seu exterior permanece em contato direto com o ar atmosférico, sendo nutrido por uma corrente fechada de meio de cultivo. O PBRL pode ser considerado um sistema recente e pouco popularizado, porém com elevado potencial produtivo e grande capacidade de mitigação de produções industriais de $\mathrm{CO}_{2}$, além de possuir colheita com elevada concentração de matéria seca, ponto crítico para alguns autores (Mata et al., 2010; Linares et al., 2012).

O desenvolvimento de novas configurações de fotobiorreatores tem por objetivo a maximização da produção de biomassa, assim como atender alguma especificidade do fim que será empregada a produção, como pode ser o caso da composição lipídica, para aplicação na produção de biocombustíveis, ou nutricional, quando empregado na alimentação animal ou fertilização de solo.

Assim como o desenvolvimento de novas configurações, alguns parâmetros de controle do cultivo se tornam fatores determinantes para esse avanço, sendo que a disponibilidade de fonte inorgânica de carbono e intensidade da radiação recebida se mostram como alguns dos principais (Borghetti, 2009; Soares et al., 2011).

Partindo destes preceitos, esse trabalho teve como objetivo verificar a influência do fornecimento artificial de $\mathrm{CO}_{2}$ e da variação da radiação fotossinteticamente ativa (RFA) na biomassa algal produzida no PBRL, avaliando a produtividade, eficiência energética e conteúdo de Nitrogênio e Fósforo. 


\section{MATERIAL E MÉTODOS}

O trabalho foi desenvolvido nas instalações do Grupo de Agroenergética, integrado ao Departamento de Produção Vegetal: Botânica e Proteção Vegetal da Escola Técnica Superior de Engenheiros Agrônomos da Universidade Politécnica de Madrid (UPM), Madri, Espanha.

A instalação utilizada foi a estufa de cultivo de microalgas, onde se encontra instalado o fotobiorreator laminar (PBRL) de cultura mista (gêneros Chlorella e Scenedesmus) patenteado pelo pesquisador Dr. Jesús Fernández sob a tutela da UPM (Fernández, 2011).

\subsection{Dados ambientais}

A estufa de cultivo possuía sistemas automatizados de mensuração da radiação fotossinteticamente ativa (RFA) solar, temperatura e concentração de $\mathrm{CO}_{2}$, com funcionamento contínuo durante as 24 horas do dia.

\subsection{Meio de cultivo}

A solução nutritiva possuía valores de $\mathrm{pH}$ iguais a 6,5 e composição química de 200 , 87,3 e $166 \mathrm{mg} \mathrm{L}^{-1}$ de nitrogênio, fósforo e potássio, respectivamente, além de boro $(0,068)$, cobre $(0,036)$, ferro $(0,5)$, magnésio $(0,25)$, molibdénio $(0,009)$ e zinco $\left(0,025 \mathrm{mg} \mathrm{L}^{-1}\right)$. A solução nutritiva foi submetida a monitoramento de macronutrientes duas vezes por semana, com análises de nitrogênio orgânico e amoniacal pelo método Kjeldhal, nitrogênio nítrico por redução prévia a nitrogênio amoniacal com liga de Devarda seguido do método Kjeldhal, fósforo por método colorimétrico de vanadato-molibdato e potássio por fotometria de chama (APHA et al., 2012).

\subsection{Desenho experimental}

A pesquisa foi realizada em dois módulos centrais do PBRL, os quais foram estratificados segundo critérios específicos a cada parâmetro analisado, $\mathrm{CO}_{2}$ e RFA:

- $\mathrm{CO}_{2}$ : para avaliar a influência do seu fornecimento, interrompeu-se o abastecimento de um dos módulos, mantendo-o sem adição artificial de $\mathrm{CO}_{2}$, exposto somente a concentração atmosférica $(0,033 \%$ volume/volume - v/v), além de manter o outro módulo com fornecimento artificial médio de $0,38 \%$ (v/v) de $\mathrm{CO}_{2}$ no interior da câmara formada entre os dois painéis.

- RFA: por haver diferença na intensidade da radiação solar recebida segundo altura e orientação, a mensuração dos valores de RFA foi realizada a partir dos dados dos sensores instalados em suportes independentes, paralelos ao PBRL, sendo três em cada orientação (oeste e leste), justificando assim a divisão da superfície do painel em três faixas (superior, mediana, inferior). Para definir os períodos fotoquímicos (luminoso) e químicos (escuro) em todos os índices (temperatura, fornecimento de $\mathrm{CO}_{2}$ e RFA), foi adotado o valor de RFA de $15 \mu \mathrm{mol} \mathrm{m} \mathrm{s}^{-1}$.

Para melhorar o tratamento estatístico, foram realizadas três subdivisões por faixa, perfazendo um total de nove blocos por superfície e, consequentemente, 18 por painel (Figura 1).

Os fotobiorreatores já se encontravam em funcionamento no início do experimento, sendo que estes haviam sido inoculados inicialmente com espécies dos gêneros Chlorella e Scenedesmus, com composição aproximada de 80 e $20 \%$, respectivamente, no momento do experimento.

A coleta e o tratamento da biomassa algal foram realizados manualmente, com intervalos semanais durante as quatro semanas de estudo.

A produtividade foi avaliada em produções de biomassa algal em matéria seca por unidade de área do fotobiorreator e dia $\left(\mathrm{g} \mathrm{m}^{-2} \mathrm{~d}^{-1}\right)$. 


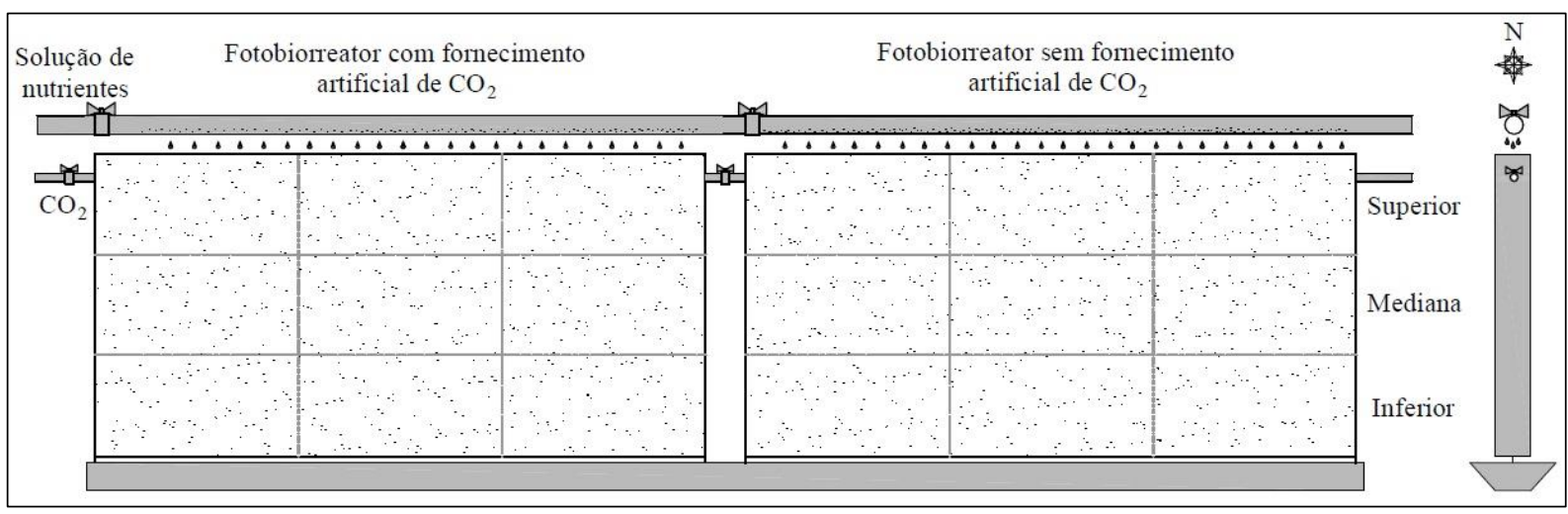

Figura 1. Esquema representativo do desenho experimental.

As análises de Nitrogênio orgânico e Fósforo total em biomassa seca foram realizadas pelo método Kjeldahl em biomassa finamente triturada e colorimétrico vanadato-molibdato nas cinzas da biomassa produzida.

Como é sabido, a energia proporcionada por cada mol de fótons no intervalo de radiação visível e aproveitável na reação fotossintética (400 a $700 \mathrm{~nm}$ ) é de 40,8 - 70,5 kcal, sendo que para determinação da eficiência energética do consórcio microalgal foi utilizado o valor médio; 55,65 kcal mol ${ }^{-1}$.

Segundo análises realizadas pelo Grupo de Agroenergética da UPM, a biomassa algal produzida nas condições descritas possui cerca de $4.200 \mathrm{kcal} \mathrm{kg}^{-1}$.

As eficiências produtivas e energéticas da atividade fotossintética das microalgas foram calculadas segundo as seguintes equações:

Eficiência produtiva $\left(\mathrm{g} \mathrm{mol}^{-1}\right)=\left[\frac{\text { Produtividade }\left(\mathrm{g} \mathrm{m}^{-2} \mathrm{~d}^{-1}\right)}{\text { Energia recebida }\left(\mathrm{mol} \mathrm{m}^{-2} \mathrm{~d}^{-1}\right)}\right]$

Eficiência energética $(\%)=\left[\frac{\text { Eficiência produtiva }\left(\mathrm{g} \mathrm{mol}^{-1}\right) \times 4,2\left(\mathrm{kcal} \mathrm{g}^{-1}\right)}{55,65 \mathrm{kcal} \mathrm{mol}^{-1}}\right] \times 100$

O processamento dos dados foi realizado por meio de análises estatística (ANOVA análise de variância para $\mathrm{p}<0,05)$ e gráfica.

Vale ressaltar que o trabalho teve discussões baseadas, em sua maioria, em cultivos puros em soluções aquosas, pois, como citado anteriormente, o PBRL se trata de um sistema recente e pouco relatado cientificamente.

\section{RESULTADOS E DISCUSSÃO}

\subsection{Dados de condições ambientais}

O monitoramento das temperaturas do ambiente e do reservatório de solução nutritiva foi realizado para garantir a manutenção das condições ambientais durante o período experimental, que proporcionaram as condições necessárias ao desenvolvimento microalgal.

Não houve grandes variações da temperatura da solução nutritiva, permanecendo entre 21,72 e $26,45^{\circ} \mathrm{C}$ na fase fotoquímica. Essa faixa de temperatura atende à temperatura tida como ideal para cultivos nestas condições, pois, segundo Chen et al. (2009), as espécies mais comuns de algas se desenvolvem entre 16 e $27^{\circ} \mathrm{C}$.

Os valores médios de radiação fotossinteticamente ativa (RFA) variaram entre 92,9 - 
173,4 e 121,1 - 191,4 $\mu \mathrm{mol} \mathrm{m} \mathrm{m}^{-2} \mathrm{~s}^{-1}$ para orientação oeste e leste, respectivamente. Em relação ao fornecimento de $\mathrm{CO}_{2}$, a concentração deste gás, fornecido artificialmente no interior do PBRL foi, em média, de $0,38 \%(\mathrm{v} / \mathrm{v})$, diferentemente do outro módulo que dispunha somente do $\mathrm{CO}_{2}$ ambiente, para o qual se registrou $0,033 \%(\mathrm{v} / \mathrm{v})$.

\subsection{Produção de biomassa}

Influência do dióxido de carbono - $\mathrm{CO}_{2}$

As produções de biomassa algal foram agrupadas em valores médios, segundo a altura, a orientação e a presença de $\mathrm{CO}_{2}$ (Tabela 1$)$.

Tabela 1. Produtividade expressa em matéria seca $\left(\mathrm{g} \mathrm{m}^{-2} \mathrm{~d}^{-1}\right)$ referente ao fornecimento de $\mathrm{CO}_{2}$, considerando radiação fotossinteticamente ativa e temperatura.

\begin{tabular}{|c|c|c|c|c|c|c|c|c|}
\hline \multirow[t]{2}{*}{ Semana } & \multirow{2}{*}{$\begin{array}{l}\text { Temperatura média } \\
\text { do meio cultivo }\left({ }^{\circ} \mathrm{C}\right)\end{array}$} & \multirow{2}{*}{ Altura } & \multicolumn{2}{|c|}{$\operatorname{RFA}\left(\mu \mathrm{mol} \mathrm{m} \mathrm{s}^{-2}\right)$} & \multicolumn{2}{|c|}{$\begin{array}{c}\text { Com incremento de } \mathrm{CO}_{2} \\
\left(\mathrm{~g} \mathrm{~m}^{-2} \mathrm{~d}^{-1}\right)\end{array}$} & \multicolumn{2}{|c|}{$\begin{array}{c}\text { Sem incremento de } \mathrm{CO}_{2} \\
\left(\mathrm{~g} \mathrm{~m}^{-2} \mathrm{~d}^{-1}\right)\end{array}$} \\
\hline & & & Leste & Oeste & Leste & Oeste & Leste & Oeste \\
\hline \multirow{3}{*}{$1^{\circ}$} & \multirow{3}{*}{21,72} & Superior & 136,99 & 177,27 & 2,04 & 2,33 & 1,80 & 2,18 \\
\hline & & Mediana & 92,83 & 118,63 & 1,83 & 1,67 & 1,52 & 1,85 \\
\hline & & Inferior & 70,14 & 101,57 & 1,39 & 1,27 & 0,87 & 1,43 \\
\hline \multirow{3}{*}{$2^{\circ}$} & \multirow{3}{*}{24,51} & Superior & 176,01 & 209,88 & 2,45 & 1,82 & 2,12 & 1,85 \\
\hline & & Mediana & 130,00 & 141,83 & 1,73 & 1,42 & 1,40 & 1,20 \\
\hline & & Inferior & 93,10 & 122,38 & 1,65 & 1,35 & 1,49 & 1,25 \\
\hline \multirow{3}{*}{$3^{\circ}$} & \multirow{3}{*}{24,81} & Superior & 187,21 & 190,36 & 2,91 & 2,46 & 1,95 & 2,20 \\
\hline & & Mediana & 147,17 & 139,67 & 2,03 & 1,93 & 1,69 & 1,65 \\
\hline & & Inferior & 103,49 & 122,88 & 1,71 & 1,75 & 1,55 & 1,45 \\
\hline \multirow{3}{*}{$4^{\circ}$} & \multirow{3}{*}{26,43} & Superior & 195,28 & 187,89 & 1,73 & 1,19 & 1,32 & 1,27 \\
\hline & & Mediana & 156,39 & 148,81 & 1,08 & 1,17 & 0,90 & 0,91 \\
\hline & & Inferior & 104,93 & 137,57 & 0,96 & 1,33 & 1,00 & 0,91 \\
\hline \multirow{3}{*}{$\begin{array}{c}\text { Média } \\
\text { total }\end{array}$} & \multirow{3}{*}{24,37} & Superior & 173,87 & 191,35 & 2,28 & 1,95 & 1,80 & 1,88 \\
\hline & & Mediana & 131,60 & 137,24 & 1,67 & 1,55 & 1,38 & 1,40 \\
\hline & & Inferior & 92,92 & 121,10 & 1,43 & 1,42 & 1,23 & 1,26 \\
\hline
\end{tabular}

Baseado na Tabela 2 pode-se afirmar que as quantidades de biomassa algal coletadas nos distintos painéis possuem diferença estatística expressiva $(\mathrm{p}<0,05)$, evidenciando a influência do fornecimento artificial de $\mathrm{CO}_{2}$ na produtividade.

Tabela 2. Análise de variância em relação à produtividade - Soma dos quadrados tipo III.

\begin{tabular}{lccccc}
\hline Fonte & Soma dos quadrados & Gl & Quadrado Médio & Razão-F & Valor-P \\
\hline Efeitos Principais & & & & & \\
A: Fornecimento de $\mathrm{CO}_{2}$ & 1,909 & 1 & 1,909 & 13,76 & 0,0003 \\
B: Altura & 10,9267 & 2 & 5,46333 & 39,39 & 0,000 \\
C: Orientação & 0,00466944 & 1 & 0,00466944 & 0,03 & 0,8547 \\
Interação & & & & & \\
AB & 0,0740722 & 2 & 0,0370361 & 0,27 & 0,7661 \\
AC & 0,2809 & 1 & 0,2809 & 2,03 & 0,1571 \\
BC & 0,0864222 & 2 & 0,0432111 & 0,31 & 0,7329 \\
ABC & 0,14735 & 2 & 0,073675 & 0,53 & 0,5892 \\
\hline Resíduos & 18,3103 & 132 & 0,138715 & & \\
Total (corrigido) & $\mathbf{3 1 , 7 3 9 4}$ & $\mathbf{1 4 3}$ & & & \\
\hline
\end{tabular}

Nota: Gl: graus de liberdade. 
Nas Tabelas 1 e 2, evidencia-se a influência do fornecimento artificial de $\mathrm{CO}_{2}$ na produtividade do cultivo (valor-p<0,05), além de expressiva influência da radiação (valor-p do fator $\mathrm{B}<0,05$ ), assim como citam outros trabalhos (Borghetti, 2009; Gonçalves et al., 2014), claramente visíveis na Figura 2 e Tabela 3, a qual mostra o incremento percentual referente ao fornecimento do gás segundo as faixas - superior, mediana e inferior.

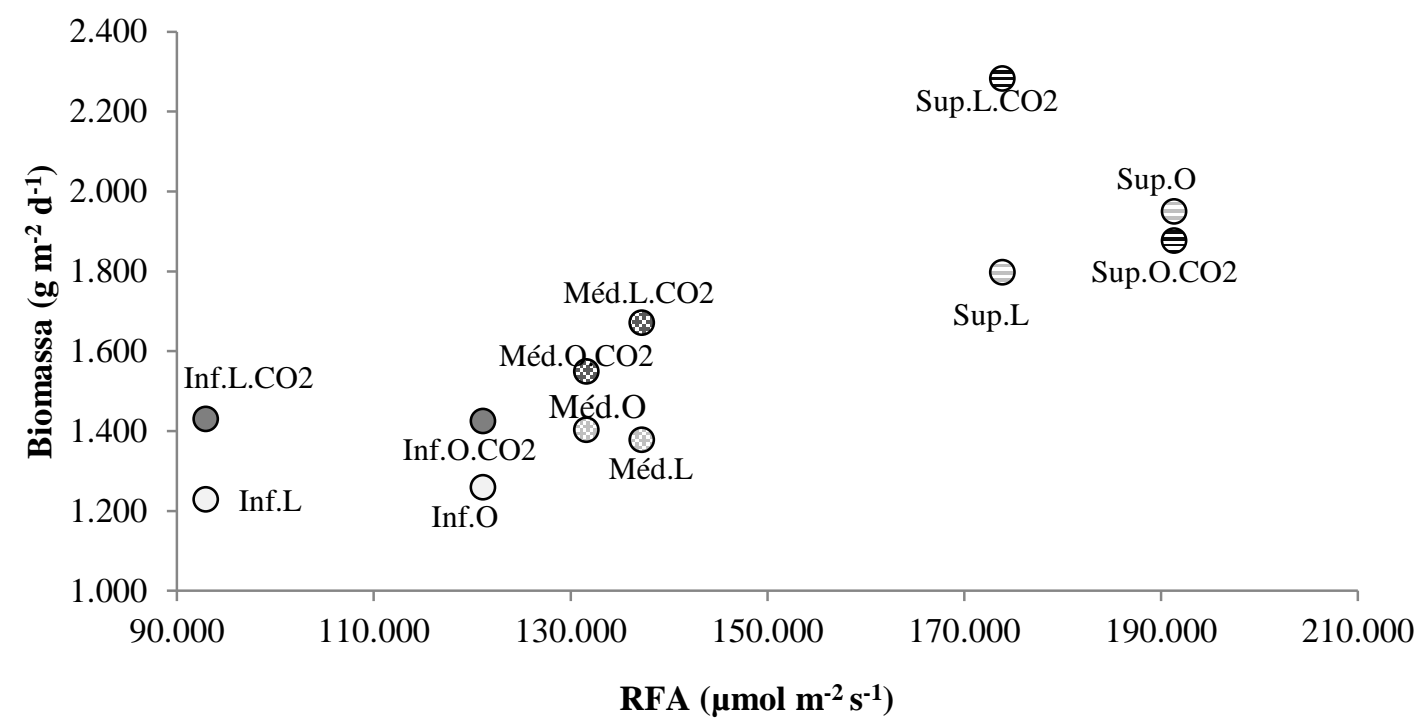

Figura 2. Produtividade média segundo influência do $\mathrm{CO}_{2}$ e Radiação.

Nota: Inf.L.CO2: faixa inferior leste com fornecimento de CO2; Inf.O.CO2: faixa inferior oeste com fornecimento de $\mathrm{CO}_{2}$; Inf.L: faixa inferior leste sem fornecimento de $\mathrm{CO}_{2}$; Inf.O: faixa inferior oeste sem fornecimento de $\mathrm{CO}_{2}$; Méd.L.CO2: faixa mediana leste com fornecimento de $\mathrm{CO}_{2}$; Méd.O.CO2: faixa mediana oeste com fornecimento de $\mathrm{CO}_{2}$; Méd.L: faixa mediana leste sem fornecimento de $\mathrm{CO}_{2}$; Méd.O: faixa mediana oeste sem fornecimento de $\mathrm{CO}_{2}$; Sup.L.CO2: faixa superior leste com fornecimento de $\mathrm{CO}_{2}$; Sup.O.CO2: faixa superior oeste com fornecimento de $\mathrm{CO}_{2}$; Sup.L: faixa superior leste sem fornecimento de $\mathrm{CO}_{2}$; Sup.O: faixa superior oeste sem fornecimento de $\mathrm{CO}_{2}$.

Tabela 3. Incremento percentual da produtividade atribuído ao $\mathrm{CO}_{2}$ segundo a altura.

\begin{tabular}{|c|c|c|c|c|}
\hline \multirow[t]{2}{*}{ Altura } & \multicolumn{2}{|c|}{ 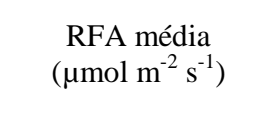 } & \multicolumn{2}{|c|}{$\begin{array}{c}\text { Incremento percentual em } \\
\mathrm{g} \mathrm{m}^{-2} \mathrm{~d}^{-1} \text { referente ao } \mathrm{CO}_{2} \\
(\%)\end{array}$} \\
\hline & Leste & Oeste & Leste & Oeste \\
\hline Superior & 173,9 & 191,4 & 27,0 & 3,9 \\
\hline Mediana & 131,6 & 137,2 & 21,3 & 10,4 \\
\hline Inferior & 92,9 & 121,1 & 16,4 & 13,2 \\
\hline
\end{tabular}

Ainda com base na Figura 2 e na Tabela 1, nota-se a inversão da variação de produção, sendo que no módulo onde se forneceu $\mathrm{CO}_{2}$, a produtividade maior foi na orientação leste, justamente o contrário do módulo que não foi submetido ao gás. Todas as faixas do módulo com fornecimento de $\mathrm{CO}_{2}$ resultaram em produções de biomassa superior, variando entre 3,9 e $27,0 \%$ de incremento sobre o módulo sem fornecimento, variações significativas segundo teste-T. Liao et al. (2014) reportaram produção de biomassa $138 \%$ maior quando fornecido $\mathrm{CO}_{2}$ em concentração de $10 \%$ (v/v) em cultivo de Chlorella pyrenoidosa. O fato do incremente inferior a literatura se deu pelas diferentes concentrações de $\mathrm{CO}_{2}$ utilizadas, como 
Yue e Chen (2005) que relataram máxima produção em concentrações de $\mathrm{CO}_{2}$ de $10 \%$ (v/v), assim como Min et al. (2012), que citaram sua máxima produtividade em concentrações de $5 \%$. O trabalho em questão teve fornecimento médio de $0,38 \%(\mathrm{v} / \mathrm{v})$, porém, vale ressaltar que, diferente da literatura citada, o trabalho se deu em cultura mista com distribuição do gás na parte interna do módulo, onde, segundo Chen et al. (2009), um incremento de concentração de $\mathrm{CO}_{2}$ superior a $5 \%$ seria tóxico a certas espécies de microalgas.

\section{Fatores que influenciam a Radiação Fotossinteticamente Ativa:}

\section{Orientação}

Ao analisar a RFA média recebida por superfície (Tabela 3), notam-se diferenças na quantidade de RFA disponível, causada pela orientação, com uma maior radiação na superfície orientada a oeste. Porém, ao relacionar esta informação aos dados de produção, não se pode afirmar a relação orientação-produção (Tabela 2), porque estatisticamente não é um fator condicionante (valor-p>0,05).

\section{Altura}

Baseando-se na análise estatística (Tabela 2), pode-se comprovar uma explícita influência da altura na produtividade (valor-p<0,05), relação esta também apreciável na Figura 1.

$\mathrm{Na}$ Tabela 4 constam os valores dos incrementos percentuais, calculados com base na faixa inferior do painel - parte menos favorecida pela radiação - seguindo gradiente crescente e ascendente no sentido vertical.

Tabela 4. Incremento percentual da produtividade e radiação, seguindo de sentido vertical com referência na faixa inferior.

\begin{tabular}{|c|c|c|c|c|c|c|c|c|c|}
\hline \multirow[t]{2}{*}{ Altura } & \multicolumn{2}{|c|}{$\begin{array}{c}\text { RFA } \\
(\%)\end{array}$} & \multicolumn{2}{|c|}{$\begin{array}{c}\text { Produção com } \\
\text { incremento de } \mathrm{CO}_{2} \\
(\%)\end{array}$} & \multicolumn{2}{|c|}{$\begin{array}{c}\text { Produção sem } \\
\text { incremento de } \mathrm{CO}_{2} \\
(\%)\end{array}$} & \multicolumn{3}{|c|}{$\begin{array}{l}\text { Média da produção } \\
(\%)\end{array}$} \\
\hline & Leste & Oeste & Leste & Oeste & Leste & Oeste & Leste & Oeste & Total \\
\hline Superior & 87,84 & 59,38 & 61,18 & 37,16 & 51,69 & 48,26 & 56,44 & 42,71 & 49,57 \\
\hline Mediana & 40,81 & 13,63 & 16,94 & 8,88 & 16,92 & 9,86 & 16,93 & 9,37 & 13,15 \\
\hline Inferior & 0,00 & 0,00 & 0,00 & 0,00 & 0,00 & 0,00 & 0,00 & 0,00 & $\mathbf{0 , 0 0}$ \\
\hline
\end{tabular}

Ao analisar as Tabelas 2 e 4, fica evidente a relação direta entre esses dois fatores (produção e radiação), caracterizando o gradiente descrito anteriormente que se inicia na parte inferior (menor disponibilidade de radiação), encontrando-se o mesmo comportamento na produção de biomassa, assim como relatam Gonçalves et al. (2014) em experimento com variação controlada da radiação em culturas puras.

Esse aumento da RFA provocou o aumento da atividade fotossintética, atingindo o máximo de saturação de luz e, consequentemente, máxima produtividade. Uma vez atingido esse índice, ocorreu saturação do sistema receptor e a produção não manteve essa relação com a RFA (Tabela 4), sofrendo declínio com o contínuo aumento da intensidade do fluxo de fótons recebidos, fato notável na Tabela 1 (valor de máxima produtividade não coincide com a máxima radiação justamente por esta haver ultrapassado o ponto de saturação máxima), provocado pela excessiva geração de oxigênio via foto-ativação, gasto energético com a dissipação deste excesso de radiação e danos no sistema fotossintético, fenômeno conhecido como fotoinibição (Benemann, 2006; Borges et al., 2007; Seo et al., 2014). 
Chang et al. (2016) avaliando o cultivo de Chlorella vulgares, em cultivo em suspensão obtiveram seu ponto de saturação máxima em intensidade de $120 \mu \mathrm{mol} \mathrm{m} \mathrm{m}^{-2} \mathrm{~s}^{-1}$, valor semelhante aos $150 \mu \mathrm{mol} \mathrm{m} \mathrm{m}^{-2} \mathrm{~s}^{-1}$ verificados por Liu et al. (2013) ao avaliar o ponto de saturação máxima de um cultivo de Scenedesmus obliquus em fotobiorreator vertical. Apesar da impossibilidade de se determinar o exato ponto de saturação máximo deste estudo, possivelmente se encontra entre 173 e $191 \mu \mathrm{mol} \mathrm{m} \mathrm{m}^{-2} \mathrm{~s}^{-1}$, intervalo que ficou clara a fotoinibição.

Com base nos dados de produtividade descritos na Tabela 1, foi calculada a influência da variação da RFA, com a diferença entre a faixa de menor e maior recepção de radiação, chegando a valores de 60,5 e 52,8\% nos módulos com fornecimento de $\mathrm{CO}_{2}$ e sem fornecimento, respectivamente. Essa maior influência da RFA no módulo com fornecimento de $\mathrm{CO}_{2}$ é justificado pela influência deste na produção, a qual já foi discutida anteriormente. Borghetti (2009) e Gonçalves et al. (2014) descrevem essas influências ao afirmar que o crescimento de microalgas está diretamente relacionado com a taxa de fixação de $\mathrm{CO}_{2} \mathrm{e}$ utilização de energia luminosa.

Min et al. (2012), avaliando a influência destes mesmos parâmetros em cultura pura e solução aquosa, também relatam essa interação, obtendo seu melhor rendimento em RFA de $100 \mu \mathrm{mol} \mathrm{m} \mathrm{m}^{-2} \mathrm{~s}^{-1}$ e $5 \%$ de $\mathrm{CO}_{2}$. Os autores observam não haver influência da radiação em ausência do fornecimento de $\mathrm{CO}_{2}$, a qual foi verificada nesse estudo, pois assim como supracitado, houve em média variação da produtividade de 52,8\% no PBRL sem fornecimento artificial de $\mathrm{CO}_{2}$. Este fato é justificado por se tratar de cultura mista, diferentemente do trabalho citado, além de ter sido desenvolvido em fotobiorreator que possibilita melhor assimilação do $\mathrm{CO}_{2}$ do ambiente, onde houve em média $0,033 \%$ de $\mathrm{CO}_{2}$.

\subsection{Concentração de nutrientes: Nitrogênio e Fósforo}

As concentrações médias de nutrientes, expressas em percentagem do elemento por unidade de massa da biomassa algal, estão dispostas na Tabela 5.

Tabela 5. Conteúdo médio de nitrogênio e fósforo na biomassa algal em função da altura, orientação e fornecimento artificial de $\mathrm{CO}_{2}$. Valores expressos em \% sobre peso seco de biomassa.

\begin{tabular}{|c|c|c|c|c|c|c|c|}
\hline \multirow{2}{*}{ Altura } & & \multicolumn{3}{|c|}{ Com incremento de $\mathrm{CO}_{2}$} & \multicolumn{3}{|c|}{ Sem incremento de $\mathrm{CO}_{2}$} \\
\hline & & Leste & Oeste & Média & Leste & Oeste & Média \\
\hline \multirow{2}{*}{ Superior } & $\% \mathrm{~N}$ & 7,369 & 7,604 & 7,487 & 7,530 & 7,458 & 7,494 \\
\hline & $\% \mathrm{P}$ & 0,844 & 0,883 & 0,864 & 0,850 & 0,977 & 0,914 \\
\hline \multirow{2}{*}{ Mediana } & $\% \mathrm{~N}$ & 7,700 & 7,821 & 7,760 & 7,750 & 7,548 & 7,649 \\
\hline & $\% \mathrm{P}$ & 0,943 & 0,827 & 0,885 & 0,783 & 0,852 & 0,818 \\
\hline \multirow{2}{*}{ Inferior } & $\% \mathrm{~N}$ & 7,692 & 7,877 & 7,784 & 7,727 & 7,607 & 7,667 \\
\hline & $\% \mathrm{P}$ & 0,762 & 0,825 & 0,794 & 0,742 & 0,894 & 0,818 \\
\hline Média N & & 7,587 & 7,767 & 7,677 & 7,669 & 7,538 & 7,603 \\
\hline Média P & & 0,850 & 0,845 & 0,847 & 0,792 & 0,908 & 0,850 \\
\hline
\end{tabular}

Com base em análise estatística, não houve diferenças significativas entre as concentrações de nitrogênio na biomassa oriunda dos distintos sistemas. Porém, com base na Tabela 6, o conteúdo de fósforo presente na biomassa algal foi evidentemente influenciado pela radiação (RFA), pois o fator orientação resultou em valor $\mathrm{p}<0,05$, além do fator altura valor $\mathrm{p}=0,05$. Ainda segundo a análise estatística (Tabela 6) o fornecimento de $\mathrm{CO}_{2}$ tem 
influência sobre a concentração de fósforo somente quando em interação com a orientação (radiação).

Tabela 6. Análise de variância referente a concentração de P.

\begin{tabular}{lccccc}
\hline Fontes & Soma dos Quadrados & Gl & Quadrados Médios & Razão-F & Valor-P \\
\hline Efeitos Principais & & & & & \\
A: Fornecimento de $\mathrm{CO}_{2}$ & $8,64648 \mathrm{E}-05$ & 1 & $8,64648 \mathrm{E}-05$ & 0,01 & 0,9224 \\
B: Altura & 0,0571021 & 2 & 0,0285511 & 3,17 & 0,0537 \\
C: Orientação & 0,0381464 & 1 & 0,0381464 & 4,24 & 0,0467 \\
Interacão & 0,0301453 & 2 & 0,0150727 & 1,68 & 0,2014 \\
AB & 0,0441108 & 1 & 0,0441108 & 4,91 & 0,0332 \\
AC & 0,0377203 & 2 & 0,0188602 & 2,1 & 0,1375 \\
BC & 0,00584734 & 2 & 0,00292367 & 0,33 & 0,7245 \\
ABC & 0,323729 & 36 & 0,00899247 & & \\
\hline Resíduos & $\mathbf{0 , 5 3 6 8 8 8}$ & $\mathbf{4 7}$ & & & \\
Total (corrigido) & & &
\end{tabular}

É sabido que o incremento da radiação favorece a fosforilação fotossintética cíclica no fotossistema I, o que conduz a formação de ATP a partir de ADP e fosfato retirado do meio nutriente. Uma fração do ATP fotossintético é utilizada na fosforilação do $\mathrm{NAD}^{+}$formando $\mathrm{NADP}^{+}$e outra parte na fosforilação dos açúcares utilizados na assimilação do carbono (ciclo de Calvin-Benson). Por outro lado, conforme Tredici (2010), a concentração de fósforo condicionada à radiação e fornecimento artificial de $\mathrm{CO}_{2}$ é possivelmente pela inter-relação entre a energia captada, seu posterior transporte pela coenzima $\mathrm{NADP}^{+}$(nicotinamida adenina dinucleótido fosfato) e subsequente redução do $\mathrm{CO}_{2}$ no ciclo de Calvin-Benson. A maior disponibilidade de radiação e $\mathrm{CO}_{2}$ estimula uma maior presença de $\mathrm{NADP}^{+}$, o que proporciona maior concentração de fósforo a biomassa submetida a estas condições.

\subsection{Eficiência do cultivo}

Verificou-se variável produção de biomassa por unidade de energia, porém, com comportamento oposto a produção por unidade de área. Torna-se evidente, principalmente ao se analisar os dados médios, que seguiram variação inversa à variação da radiação. Isso é justificável pela teoria da fotoinibição descrita anteriormente, pois se em presença de grande quantidade de fótons os centros fotossintéticos não são capazes de aproveitá-los todos, perdendo eficiência, a recepção de um pequeno fluxo de fótons traz o aproveitamento máximo destes, consequentemente, permitindo máxima eficiência por unidade energética.

Tratando-se unicamente da RFA, os valores médios variaram de 0,29 a $0,44 \mathrm{~g} \mathrm{~mol}^{-1}$ para o módulo com fornecimento de $\mathrm{CO}_{2}$ e 0,28 a $0,38 \mathrm{~g} \mathrm{~mol}^{-1}$ para o módulo sem fornecimento de $\mathrm{CO}_{2}$.

Quando se isola o fornecimento de $\mathrm{CO}_{2}$ como único condicionante, chega-se a valores médios de 0,35 e $0,31 \mathrm{~g} \mathrm{~mol}^{-1}$ para o módulo que houve fornecimento de $\mathrm{CO}_{2}$ e sem fornecimento, respectivamente.

A eficiência energética da atividade fotossintética do consórcio microalgal se manteve entre 2,12 e 3,32\%, valores estes que se enquadram no intervalo descrito por Stephens et al. (2010) entre 1 e $4 \%$, porém, são sutilmente superiores ao intervalo de 2 a $3 \%$ citado pela literatura (Benemann, 2006; Pruvost et al., 2016) para sistemas abertos e externos.

Ozkan et al. (2012) reportaram 2,02\% de eficiência fotossintética ao avaliarem Botryococcus braunii em fotobiorreator de cultivo imobilizado em biofilme. Ao avaliar a eficiência fotossintética de Chlorella pyrenoidosa cultivadas em fotobiorreator tubular, Liao et al. (2014) lograram valores de 1,15 e 2,92 para concentrações de $\mathrm{CO}_{2}$ de 0,03 e $10 \%$, respectivamente. Valores esses levemente inferiores aos obtidos neste estudo. 


\section{CONCLUSÕES}

$\mathrm{O}$ fornecimento artificial de $\mathrm{CO}_{2}$ e a radiação fotossinteticamente ativa, nas condições deste estudo, se mostraram representativamente influente na produção e no percentual de fósforo da biomassa.

$\mathrm{O}$ fornecimento de $0,38 \%$ de $\mathrm{CO}_{2}$, associado à variação de RFA de 92,92 a $191,35 \mu \mathrm{mol} \mathrm{m} \mathrm{s}^{-1}$ aumentou a produtividade em até $60,5 \%$ a produção de biomassa.

Consequentemente, as eficiências produtivas e energéticas da atividade fotossintética também se mostraram influenciadas, variando entre 0,28 e $0,44 \mathrm{~g} \mathrm{~mol}^{-1}$ e 2,12 a $3,32 \%$, respectivamente.

\section{AGRADECIMENTOS}

Ao CNPq, pela bolsa Ciência sem fronteiras, Processo N. 214762/2012-2, à Universidade de Uberaba e a todos que compõe o Grupo de Agroenergética, em especial ao Professor Dr. Jesús Fernández.

\section{REFERÊNCIAS}

AMERICAN PUBLIC HEALTH ASSOCIATION - APHA; AMERICAN WATER WORKS ASSOCIATION - AWWA; WATER ENVIRONMENT FEDERATION - WEF. Standard methods for the examination of water and wastewater. 22nd edition. Washington, D.C., 2012.724p.

BENEMANN, J. R. Systems and economic analysis of microalgae ponds for conversion of $\mathrm{CO}_{2}$ to biomass. Pittsburgh: Department of Energy, 2006. 215p.

BORGES, L.; FARIA, B. M.; ODEBRECHT, C. ABREU, P. C. Potencial de absorção de carbono por espécies de microalgas usadas na aquicultura: Primeiros passos para o desenvolvimento de um "mecanismo de desenvolvimento limpo". Atlântica, v. 29, p. 35-46, 2007.

BORGHETTI, I. A. Avaliação do crescimento da microalga Chlorella minutissima em meio de cultura com diferentes concentrações de manipuera. 2009. $103 \mathrm{f}$. Dissertação (Mestrado em Processos Biotecnológicos) - Universidade Federal do Paraná, Curitiba, 2009.

CHANG, H. X.; HUANG, Y.; FU, Q.; LIAO, Q.; ZHU, X. Kinetic characteristics and modeling of microalgae Chlorella vulgares growth and $\mathrm{CO}_{2}$ biofixation considering he coupled effects of light intensity and dissolved inorganic carbon. Bioresource Technology, v. 206, p. 231-238, 2016. http://dx.doi.org/10.1016/j.biortech.2016.01.087

CHEN, P.; MIN, M.; CHEN, Y.; WANG, L.; LI, Y.; CHEN, Q. et al. Review of the biological and engineering aspects of algae to fuels approach. International Journal of Agriculture and Biological Engineering, v. 2, p. 1-30, 2009.

FERNÁNDEZ, J. Laminar photobioreactor for the production of microalgae. U.S. Patent Application 13/695,709, 7 Abr. 2011.

GONÇALVES, A. L.; SIMÕES, M.; PIRES, J. C. M. The effect of light supply on microalgal growth, $\mathrm{CO}_{2}$ uptake and nutrient removal from wastewater. Energy Conversion and Management, v. 85, p. 530-536, 2014.

http://dx.doi.org/10.1016/j.enconman.2014.05.085 
LIAO, Q.; LI, L.; CHEN, R.; ZHU, X. A novel photobioreactor generating the light/dark cycle to improve microalgae cultivation. Bioresource Technology, v.161, p.186-191. 2014. http://doi.org/10.1016/j.biortech.2014.02.119

LINARES, L. C. F.; MONTOYA, J. M.; OROPEZA, A. M.; CORONA, J. A. B. Producción de biocombustibles a partir de microalgas. Ra Ximhai, v.8, p.101-105. 2012.

LIU, T.; WANG, J.; HU, Q.; HU, Q.; CHENG, P.; JI, B. et al. Attached cultivation technology of microalgae for efficient biomass feedstock production. Bioresource Technology, v. 127, p. 216-222, 2013. http://dx.doi.org/10.1016/j.biortech.2012.09.100

MATA, T. M.; MARTINS, A. A.; CAETANO, N. S. Microalgae for biodiesel production and other applications: A review. Renewable and Sustainable Energy Reviews, v. 14, p. 217-232, 2010. http://dx.doi.org/10.1016/j.rser.2009.07.020

MIN, M.; HU, B.; ZHOU, W.; LI, Y.; CHEN, P.; RUAN, R. Mutual influence of light and $\mathrm{CO} 2$ on carbon sequestration via cultivating mixotrophic alga Auxenochlorella protothecoides UMN280 in an organic carbon-rich wastewater. Journal of Applied Phycology, v. 24, p. 1099-1105, 2012. http://dx.doi.org/10.1007/s10811-011-9739-3

OZKAN, A.; KINNEY, K.; KATZ, L.; BERBEROGLU, H. Reduction of water and energy requirement of algae cultivation using an algae biofilm photobioreactor. Bioresource Technology, v. 114, p. 542-548, 2012. http://dx.doi.org/10.1016/j.biortech.2012.03.055

PRIYADARSHANI, I.; RATH, B. Commercial and industrial applications of micro algae - A review. Journal of Algal Biomass Utilization, v. 3, p. 89-100, 2012.

PRUVOST, J.; LE GOUIC, B.; LEPINE, O.; LEGRAND, J.; LE BORGNE, F. Microalgae culture in buildind-integrated photobioreactors: Biomass production modeling and energetic analysis. Chemical Engineering Journal, v. 284, p. 850-861, 2016. http://dx.doi.org/10.1016/j.cej.2015.08.118

SEO, Y. H.; CHO, C.; LEE, J. Y.; HAN, J. I. Enhancement of growth and lipid production from microalgae using fluorescente paint under the solar radiation. Bioresource Technology, v. 173, p. 193-197, 2014. http://dx.doi.org/10.1016/j.biortech.2014.09.012

SOARES, A. H.; TORBEY, B. I.; PASSOS, F. B.; MONTEIRO, L. P. C. Técnicas de crescimento da microalga Dunaliella salina para produção de biodiesel e separação de óleo e biomassa produzido no processo. Engevista, v. 13, p. 102-110, 2011.

STEPHENS, E.; ROSS, I. L.; MUSSGNUG, J. H.; WAGNER, L. D.; BOROWITZKA, M. A.; POSTEN, C. et al. Future prospects of microalgal biofuel production systems. Trends in Plant Science, v. 15, p. 554-564, 2010. http://dx.doi.org/10.1016/j.tplants.2010.06.003

TREDICI, M. R. Photobiology of microalgae mass cultures: understanding the tools for the next green revolution. Biofuels, v. 1, p. 143-162, 2010.

http://dx.doi.org/10.4155/bfs.09.10

YUE, L.; CHEN, W. Isolation and determination of cultural characteristics of a new highly $\mathrm{CO}_{2}$ tolerant fresh water microalgae. Energy Conversion and Management, v. 46, p. 1868-1876, 2005. http://dx.doi.org/10.1016/j.enconman.2004.10.010 